\title{
Géometrie Et Arts-Graphiques Pour Les Pprentissages Fondamentaux A L'ecole Elementaire
}

\author{
Docteur Kadjo Koutou \\ Enseignant-Chercheur, Ecole Normale Supérieure, \\ Abidjan, Côte d'Ivoire
}

Doi: 10.19044/esj.2017.v13n28p321 URL:http://dx.doi.org/10.19044/esj.2017.v13n28p321

\begin{abstract}
Geometric activities are a great way for children, especially those in elementary school, to enter into the mathematical world. To handle, discover, invent, recognize, and identify progressively geometric forms, the child is required to play in space and time with multiple tools and material in order to create new and representative forms. The results obtained have high performance and it helps the teacher to achieve some objectives in all domain of learning, and in particular in the acquisition of transversal skills such as writing and reading. Our suggestions set out ways of improving the objectives set in relation to the undeniable importance of the use of geometric shapes in the practice of graphic art in elementary schools.
\end{abstract}

Keywords: Geometric, graphic art, fundamental learning, elementary school

\section{Resume}

Les activités géométriques sont pour les enfants et plus particulièrement ceux de l'école élémentaire, un excellent moyen d'entrer dans le monde mathématique. Outre, le plaisir de manipuler, de découvrir, d'inventer, de reconnaître et d'identifier progressivement des formes géométriques, l'enfant va jouer dans l'espace et dans le temps, avec de multiples matériels et matériaux à l'effet de créer des formes nouvelles et représentatives. Les résultats enregistrés sont performants, pouvant aider l'enseignant à atteindre un certain nombred'objectifs dans tous les domaines de l'apprentissage, et notamment dans l'acquisition des compétences transversales que sont l'écriture et la lecture. Nos suggestions tracent des voies relatives à l'amélioration des objectifs fixés par rapport à l'importance indéniable de l'utilisation des formes géométriques dans la pratique des artsgraphiques à l'école élémentaire. 
Mots-clés: Géométrie, artsgraphiques, apprentissage fondamental, école élémentaire

\section{Introduction}

Les arts graphiques définis comme création de belles formes sur un support est une «plate-forme» de facteurs artistiques et esthétiques où la géométrie s'est imposée véritablement comme le complément indispensable. Rien ne se fait au niveau du graphisme à l'école élémentaire en dehors des formes géométriques. Tout est géométrie : toute forme, tout objet est décrit ou s'inscrit dans une forme géométrique. Partout, autour de nous, l'on observe des tracés symboliques de formes carrées, triangulaires ou circulaires et leurs dérivés et de différentes lignes, tant dans l'espace que dans le temps.

La géométrie étudie les figures du plan et de 1'espace. Elle demeure dans l'expression de toute chose et dans l'activité grapho-motrice au niveau de l'enfant.Du restedans la formation de celui-ci, elle participe efficacement au développement de l'apprenant dans l'apprentissage de l'écriture et de la lecture à travers la variété de son graphisme.

La géométrie, outil fondamental de l'école maternelle, se redécouvre au niveau de l'école élémentaire dans la quasi-précision de ses formes et ordonne à l'instinct créatif de l'enfant de se baser sur les notions des arts graphiques pour développer ses acquis. C'est sous cet angle qu'Apollinaire (1967) écrivait : «La géométrie est aux arts plastiques, ce que la grammaire est à l'art de l'écrivain». L'idée de cet auteur souligne la cohésion extrême et l'indispensabilité de la géométrie dans la pratique des arts graphiques et son rôle éminent dans certains apprentissages fondamentaux à l'école élémentaire.

Dans cette étude nous allons montrer de manièrethéorique et pratique, les facteurs géométriques qui contribuent à la corrélation et à l'interaction des deux disciplines dans les apprentissages fondamentaux : l'écriture et la lecture à l'école élémentaire.

\section{Quelques considerations theoriques}

«En peinture, les lignes droites évoquent la stabilité ; elles découpent l'espace intérieur du tableau et mettent en valeur le format de l'image. Les lignes courbent traduisent l'agitation et le déséquilibre; elles créent une relation dynamique et mouvementée avec l'ordre statique et régulier du cadre. La confrontation entre ces deux réseaux graphiques crée dans l'œil du spectateur une instabilité et une tension qui "agitent" l'image», écrivaient Giboulet et Barilleau (1997).

En effet, la géométrie occupe une place privilégiée dans l'enseignement des mathématiques. De nombreuses études pédagogiques 
prouvent son intérêt, car elle permet aux élèves de développer une réflexion sur des problèmes divers, de visualiser des figures du plan et de l'espace, de rédiger des démonstrations, de déduire des résultats d'hypothèses énoncées. Mais plus encore, «le raisonnement géométrique est beaucoup plus riche que la simple déduction formelle, car il s'appuie sur l'intuition née de l'observation des figures», cite Tiémoko (2017).

Selon Madou (2017), «les activités géométriques sont pour les enfants et plus particulièrement ceux de l'école élémentaire, un excellent moyen d'entrer dans le monde mathématique. Outre, le plaisir de manipuler, de découvrir, de reconnaître et d'identifier progressivement des formes géométriques, l'enfant va jouer dans l'espace et dans le temps, avec de multiples matériels et matériaux à l'effet de créer des formes représentativesnouvelles. Ces activitésrécréatives permettent à l'enseignant d'atteindre un certain nombred'objectifs dans tous les domaines d'apprentissage».

Pour Giboulet et Barilleau (1997), «le carré, le cercle et le triangle sont les trois formes géométriques primaires qui participent à la configuration des formes représentatives. Les autres formes géométriques plus complexes dérivent de ces trois figures. Ainsi, elles permettent de simplifier un motif, de fragmenter sa surface et de mettre en valeur le tracé régulateur de soncontour (symétrie, rayonnement)».

N'goli (2017), soutient que «la géométrie joue un rôle important dans la pratique des arts graphiques, d'autant plus que les activités d'expression, de création, de récréation, d'initiation, de mimétisme et de logique conduites au cours des apprentissages fondamentaux par le biais du graphisme, favorisent chez l'enfant, le développement de son intelligence, de son équilibre psychologique, de sa concentration, de sa finesse dans le travail gestuel, de sa recherche de l'esthétique, de son agilité corporelle et toutes choses qui devront préparer cet apprenant à un bon parcours scolaire concourant à son bien-être social».

Malgré leur pertinence, une limite majeure apparaît, exprimée par Sékongo (2017), «qui pense que certes, le graphisme utilise des enchaînements de lignes simples, rectilignes ou courbes, continues ou discontinues et des alternances de couleurs qui se rythment et se structurent en motifs, ces activités aident l'enfant à construire ses habiletés perceptives et motrices, à développer ses compétences utiles pour la maîtrise du geste d'écriture. Cependant, elles ne constituent pas des activités préparatoires à l'écriture au sens strict car il n'y a pas de continuité directe avec l'écriture».

Pour nous, les gestes élémentaires se constituent progressivement, au fur et à mesure que la motricité générale de l'enfant se développe. Ils sont d'autant mieux maîtrisés qu'il peut les verbaliser. L'adulte accompagne les enfants pour leur faire percevoir la relation entre le geste produit et les effets 
produits. Cependant, lorsque l'enfant prend conscience de sa capacité à influencer et à contrôler sa trace, il peut la faire évoluer.

On aurait tendance à croire que la géométrie et les arts graphiques sont des disciplines très divergentes, mais au regard de leur contribution aux apprentissages fondamentaux, on s'aperçoit que leurs champs d'investigation et leurs approches présentent des similarités. La géométrie des formes et les arts graphiques essaient de représenter le monde, chacun avec les outils qui lui sont propres. Les Arts et les sciences exactes sont deux entités en quête d'une explication, et toutes deux porteuses d'interrogation.

Sous cet angle, les uns expriment leur ressenti à partir d'outils plastiques, les autres tentent de répondre en utilisant des outils conventuels et des formes abstraites. Ils se rejoignent sur un plan très important que constitue l'intuition qui est un élément essentiel dans leurs démarches respectives. Le mathématicien a besoin d'assez d'intuition pour faire avancer sa recherche et l'artiste utilise également son intuition dans sa création plastique.

Définitions et Objectifs

\section{Définitions}

Au niveau conceptuel, la géométrie est la science de la mesure du terrain. Elle est la partie des mathématiques qui étudie les figures du plan et de l'espace, selon LeGrandLarousse encyclopédique, 1978.

\section{Les artsplastiques}

Selon le Dictionnaire Hachette (1992), l'art est l'activité humaine qui aboutit à la création d'œuvre d'art dans le domaine plastique ou musical. C'est aussi chacune des activités dans lesquelles les facultés créatrices de l'homme peuvent exprimer un idéal esthétique. Le terme plastique est polysémique et de ce fait obéit à une pluralité de définitions :

- $\quad$ Toujours selon le Dictionnaire Hachette (1992), en tant qu'adjectif, est plastique ce qui a rapport aux formes, à leur harmonie et à leurs techniques. C'est aussi l'art de donner forme à une substance.

- $\quad$ En tant que nom masculin, il désigne tantôt une substance malléable, modelable ou une matière de synthèse fabriquée à partir des dérivées du pétrole ou du charbon.

Selon Galizot cité par Toraille (1962), «par Arts-plastiques, il faut entendre toutes les activités artistiques s'appliquant à l'élaboration d'images planes ou en volumes, fixes ou mobiles». Les Arts-plastiques sont en fait un moyen d'expression et de communication dont le but est de reproduire ou d'élaborer des formes abstraites ou figuratives. 


\section{Les artsgraphiques}

Les arts graphiques désignent l'ensemble des processus propres à la conception visuelle et à la mise en scène d'une création artistique, utilisant différentes techniques (écriture, typographie, dessin, peinture, gravure et estampe, photographie, etc.), cette création pouvant être utilisée à des fins uniquement artistiques, industrielles ou commerciales (messages publicitaires, édition, affiches, revues, etc.). Le graphisme est l'élément essentiel, le caractère expressif et personnel, l'écriture et la signature propre de tout projet graphique ou publicitaire. Dans les artsgraphiques, le dessin et l'utilisation des formes géométriques sont indispensables pour la conception d'une œuvre graphique.

\section{Le graphisme}

Le graphisme est une discipline qui consiste à créer, choisir et utiliser des éléments graphiques (caractères typographiques, photos, couleurs, etc.), pour élaborer un objet de communication et/ou de culture. C'est une manière propre de représenter, d'écrire, de former les mots, la disposition plastique volontaire ou involontaire de créer son style de caractère. Le graphisme est donc une manière d'écrire, de tracer des différentes lignes, souvent envisagées d'un point de vue esthétique. Le graphisme se rapporte aussi au traitement formel des informations dans une publicité (illustration, affiche, communication d'entreprise, presse, édition, design web, signalétique, identité visuelle, etc.).

\section{L'apprentissage}

Selon le Dictionnaire Hachette (1992), l'apprentissage est un ensemble de mécanisme menant à l'acquisition de savoir-faire, de savoirs ou de connaissances. C'est aussi, un ensemble de processus de mécanisation mis en œuvre par l'animal ou l'homme pour élaborer ou modifier les schèmes comportementaux spécifiques sous l'influence de son environnement et de son expérience.

L'acteur principal de l'apprentissage est appelé apprenant. On peut opposer l'apprentissage à l'enseignement dont le but est de dispenser des connaissances et des savoirs, l'acteur principal de l'enseignement étant l'enseignant, l'apprentissage consiste donc à acquérir ou à modifier une représentation d'un environnement de façon à permettre avec celui-ci des interactions efficaces ou de plus en plus efficaces.

\section{Notion de "Fondamental"}

Selon le Dictionnaire Larousse (1985), c'est ce qui sert de base, de fond, de fondement. La chose fondamentale a un caractère essentiel, 
déterminant par rapport à d'autres choses. Se dit aussi de ce qui précède le commencement, l'origine de toute chose qui est au fondement d'une telle.

\section{La lecture}

Selon le Dictionnaire Larousse encyclopédique, 1978, la lecture peut être définie comme une activité psychosensorielle qui vise à donner un sens à des signes graphiques recueillis par la vision et qui implique à la fois des traitements perceptifs et cognitifs, C'est l'action de lire, de déchiffrer toute espèce de notation, de prendre connaissance d'un texte.

L'efficacité de la lecture dépend de deux voies de traitement de l'information qui coexistent et se complètent mutuellement : l'identification des signes ou mots écrits et l'accès au son de ces éléments. Si l'un des mécanismes est déficient, un trouble de la lecture s'ensuivra.

\section{L'écriture}

Selon le Dictionnaire Hachette (1992), l'écriture est un système de signes graphiques servant à noter un message oral afin de pouvoir le conserver ou le transmettre. L'écriture est un moyen de communication qui représente le langage à travers l'inscription de signes sur des supports variés. C'est une technologie qui s'appuie sur les mêmes structures que la parole, comme le vocabulaire, la grammaire et la sémantique, mais avec des contraintes additionnelles liées au système de graphies propres à chaque langue.

\section{Objectifs}

Cette étude vise trois objectifs spécifiques:

- $\quad$ Etablir un lien entre le graphisme et la géométrie;

- Décrire les différents mécanismes utilisés dans l'apprentissage de l'écriture et celui de la lecture avec comme apport les formes géométriques;

Expliquer 1'interdisciplinarité des artsgraphiques.

\section{Présentation des différents facteurs}

\section{La Géométrie}

La géométrie classique englobe principalement la géométrie euclidienne qui est l'étude de l'espace usuel avec les notions de distance et d'angle, la géométrie affine étudie singulièrement les points et les droites, la géométrie projective ajoute aux espaces de la géométrie affine, des points à l'infini, la géométrie non euclidienne est une variante de la géométrie euclidienne et n'en diffère que par la modification de l'énoncé du cinquième postulat d'Euclide.Cette géométrie est contraire à l'intuition usuelle. Elle comprend la géométrie hyperbolique, la géométrie elliptique et la géométrie 
sphérique. Ces géométries sont encore dites classiques (In Dictionnaire encyclopédique, 1978).

Les géométries ci-dessus peuvent être généralisées en faisant varier la dimension des espaces, en changeant le corps des scalaires, c'est-à-dire utiliser des droites différentes de la droite principale ou en donnant une courbure à l'espace. Selon Poincaré, «l'espace de la géométrie possède les propriétés suivantes:

- Continu,

- Infini,

- A trois dimensions,

- $\quad$ Homogène, c'est-à-dire que tous ses points sont identiques entre eux.

- $\quad$ Isotrope, c'est-à-dire que toutes les droites qui passent par un même point sont identiques entre elles» (in Henri Poincaré, l'espace et la géométrie, Etienne Ghys, 2012).

\section{Les artsplastiques}

Le champ d'application des arts-plastiques est tellement vaste que l'on ne saurait dresser une liste exhaustive de toutes les activités qu'ils englobent. Toutefois, elles ont toutes une caractéristique commune, l'image qui est fixe ou mobile, plane ou en volume. Les arts-plastiques regroupent toutes les pratiques ou activités donnant une représentation artistique, esthétique ou poétique, au travers des formes et des volumes. Ils donnent également forme à une surface ou à un objet pour créer la beauté. Les artsplastiques s'identifient à plusieurs domaines à savoir le dessin, la peinture, l'architecture, la sculpture, la céramique, la décoration, la gravure, la communication visuelle, les arts-graphiques, la photographie, etc.

\section{L'apprentissage à l'école élémentaire}

Pour la psychologie béhavioriste, l'apprentissage est vu comme la mise en relation entre un événement provoqué par l'extérieur (stimulus) et une réaction adéquate du sujet qui traduit un changement de comportement persistant, mesurable et spécifique ou permet à l'individu de formuler une nouvelle construction mentale ou réviser une construction mentale préalable.

L'apprentissage est un concept important étudié par la psychologie du développement qui embrasse également les changements, les acquisitions et la perte de la vie embryonnaire à la mort.

L'historien Ariès, «insiste sur l'importance qu'il convient d'attribuer à l'apprentissage. Il force les enfants à vivre au milieu des adultes qui leur communiquent ainsi le savoir-faire et le savoir-vivre. Le mélange des âges qu'il entraîne lui paraît un des traits dominants de notre société, du milieu du Moyen Age au XVIIe siècle»,,(in L'enfant et la vie familiale sous l'ancien régime, Ariès Philippe, 1975). 
La formation dispensée dans les écoles élémentaires assure l'acquisition des instruments fondamentaux de la connaissance : expressions orale et écrite, lecture, calcul et résolution de problèmes, l'école élémentaire accueillant les enfants de 6 à 11 ans sur deux cycles (cycle 2 et début du cycle 3 ) et cinq niveaux de classes : le cours préparatoire 1 et 2 , le cours élémentaire 1 et 2 , le cours moyen 1 et 2 assure donc la formation et l'éducation de ces apprenants.

\section{L'écriture à l'école élémentaire}

L'écriture est la reproduction puis la production de mots, de textes selon les codes et les règles de la langue écrite, pour donner du sens et pour communiquer. L'écriture nécessite un apprentissage spécifique et il revient donc à l'enseignant de mettre en place de réelles situations d'enseignement pratique de l'écriture. L'enfant aura à s'approprier une technique ainsi qu'un objet linguistique aux enjeux scolaires et sociaux. C'est un système qui fait partie de celui plus vaste de la langue écrite, laquelle inclut la lecture. Lecture et écriture sont deux mamelles qui partagent des éléments d'un même code.

Dans les sociétés humaines émergeantes, le développement de l'écriture est probablement lié à des exigences pragmatiques comme l'échange d'informations, la tenue de comptes financiers, la codification des lois et l'enregistrement de l'histoire.

\section{La lecture dans l'apprentissage}

Un enfant de CE1, par exemple doit pouvoir copier lui-même ses exercices de français, de mathématiques, de sciences, etc. Dans le cas de certains exercices systématiques comme la grammaire, il vaut mieux donner moins de phrases à faire. Dans le cas contraire, il faut faire rédiger le texte entier par les enfants eux-mêmes. Ainsi, ils apprennent à lier systématiquement écriture et sens, dans un travail en continuité. Au cours moyen, la lecture et l'écriture sont devenues courantes et n'ont plus besoin d'être travaillées en tant que telles, mais peuvent être mises au service de tous les apprentissages: littérature, étude de la langue, mathématiques, histoire, géographie, sciences, arts, etc.

\section{Méthodes d'enquêtes}

Selon Grawitz (1993), «la méthodologie est un ensemble concerté d'opérations mises en œuvre pour atteindre un ou plusieurs objectifs. C'est un corps de principes présidant à toute recherche organisée, un ensemble de procédés employés soit pour confirmer ou pour infirmer une hypothèse».

Sous cet angle, nous avons procédé à des enquêtes de longue durée, de 1997 à 2006dans toutes les écoles élémentaires de la commune de 
Cocody, auprès des inspecteurs de l'enseignement préscolaire et primaire ainsi que de conseillers pédagogiques. L'enquête s'est étendue à des enseignants tenant une classe. Des expériences ont été réalisées avec les enfants des classes visitées au cours de ces années d'investigation.

A la fin de ces années de travail, plus précisément, en 2006, les résultats obtenus ont contribué à l'instauration des écoles maternelles dans toutes les écoles primaires au niveau national. Pour cette rentrée scolaire 2016-2017, nous avons poursuivi nos enquêtes auprès des professeurs stagiaires de Lycée en mathématiques. Un travail de recherche est fait par ces stagiaires qui ont donné un contenu vérifiant ainsi nos hypothèses. Notre échantillon portait sur un total de 120 personnes.

Pour mener à bien ce travail de recherche, nous avons, sur le plan méthodologique, fondé dans un premier temps notre collecte d'information sur une recherche bibliographique et cela dans le but de confronter un certain nombre de réflexions déjà faites sur le sujet. Notre seconde démarche s'est appuyée sur les différentes enquêtes sur le terrain qui nous ont permis de nous adresser aux strates des personnels suscités. La troisième démarche consistait pour nous, à observer les enseignants de l'école élémentaire et les professeurs stagiaires de mathématique en situation de classe.

Après toutes ces investigations, nous avons opté pour l'examen des enquêtes, l'analyse des données dans le souci de vérifier les différentes interrogations que nous nous sommes posé dès le départ.

\section{Résultats}

Les artsgraphiques ont toujours été, dans le temps et dans l'espace, l'un des outils privilégiés de la communication entre les hommes, donc un des foyers de focalisation de la communicabilité et de la sociabilité. Dans son approche conceptuelle, nous définissons la notion de graphisme comme étant une manière de tracer un trait, considéré sous l'angle esthétique, synonyme de graphie, c'est-à-dire d'écriture.

\section{Graphisme dans l'apprentissage de l'enfant}

Le graphisme et l'écriture sont des activités différentes. Cependant elles sont toutes deux de nature grapho-motriceet les tracés qui en découlent nécessitent l'usage d'un outil scripteur, une surface et un support de papier pour leur pratique. Le graphisme nécessite également, une étude, une production, unereproductionde différentes lignes, de motifs, de formes et l'exploration d'organisations spatiales pour construire des habiletés perceptives et motrices.

Les activités du graphisme peuvent impliquer implicitement des caractéristiques de l'écriture qui s'apparentent aux formes dès le début du gribouillage de l'enfance (début du dessin enfantin), et tenant compte des 
contingences spatiales, des rythmes d'espacements, de l'horizontalité de la ligne, etc. Leur transfert dans l'écriture ne se réalisera que moyennant un réinvestissement successif d'activité en activité jusqu'à ce que le lien avec l'écriture puisse émerger et être explicite. Le transfert ne peut pas être spontané. Dans tous les cas, les situations d'apprentissage doivent s'inscrire dans un vécu culturel commun et sont préférables aux exercices formels proposés sous forme de fiches.

Selon Ouamien (2017), «l'apprentissage du graphisme nécessite souvent des enchaînements de lignes simples, rectilignes ou courbes, continues ou brisées, qui se structurent parfois en motifs et jouent sur la répétition, l'alternance, les rythmes ou les multiples facettes de la symétrie. Les arts visuels, les arts décoratifs et les objets du patrimoine choisis pour leur richesse graphique, sont des ressources à privilégier. Les répertoires de motifs contribuent à renforcer les références collectives».

Ainsi, nous réaffirmons que les activités d'expression, de création, de recréation, d'initiation et de logique conduites au cours des apprentissages par le biais du graphisme favorisent chez l'enfant, l'intelligence, l'équilibre psychologique, la concentration, la finesse dans le travail, la recherche de l'esthétique, l'agilité corporelle, toutes choses qui devront préparer l'apprenant à un bon cursus scolaire.

\section{Dessin dans l'apprentissage de l'enfant}

Le dessin est l'un des éléments essentiels constituant les Activités d'Expression et de Création. Il permet la représentation d'objets, de paysages, de personnes, etc. Il traduit les pensées, les sentiments, les sensations grâce à quelques coups de pinceau ou de crayons. S'il n'est pas aussi précis que la photographie, il offre du moins l'avantage d'être un art vivant, souple et complet.

L'école encourage surtout la pratique du dessin libre et spontané qui renforce chez l'enfant la construction de soi, le développement de la personnalité. Le dessin est considéré souvent comme une activité ludique et motrice, cependant, il développe le rapport entre le geste et la trace, il possède une fonction langagière et plastique.

Selon Lagoutte (1990), «le dessin est l'une des activités qui plaisent le plus souvent aux enfants. L'enfant de l'école élémentaire a besoin de traduire ses préoccupations et d'expliquer ses intérêts de manière authentique. Il parvient à s'impliquer dans une véritable démarche de création artistique utilisant un mode de la pensée fondamentale qu'on a trop souvent laissée pour compte : le dessin».Rigaud (1993), ne demandait-il pas à ce que les enfants puissent être initiés à l'art pendant le temps scolaire et hors du temps scolaire à ce qu'ils se déscolarisent, l'image de l'art doit être recherchée». 
Le dessin et les compositions plastiques (fabrication d'objets) sont les moyens d'expression privilégiés pour les enfants de pouvoir extérioriser leur «moi». Le dessin leur permet égalementd'expérimenter les divers instruments, supports et procédés de représentation graphique. Ils découvrent, utilisent et réalisent des images et des objets de nature variée suivant les matériels et matériaux disponibles tels que, la peinture, les papiers collés, le collage en relief, les assemblages ou le modelage.

\section{Activités géométriques}

Les activités géométriques permettent à l'enseignant d'atteindre de nombreux objectifs dans tous les domaines d'apprentissage. Les artsgraphiques sont l'un des moyens les plus naturels, par le jeu pour intéresser les enfants à la géométrie. En effet, la découverte des formes commence dès la petite section de l'école maternelle et se développe à l'école élémentaire. Elle peut être envisagée à travers l'observation de tableaux d'artistes. Aborder l'art sous l'angle de la géométrie ou aborder la géométrie à travers les œuvres d'art, permet aux enfants d'obtenir des réalisations solidement construites, sobres et rigoureuses.

Les activités géométriques entretiennent de nombreux liens avec les autres domaines d'apprentissage: elles nourrissent la curiosité dans la découverte du monde, permettent à l'enfant d'exercer sa motricité, l'encouragent à exprimer des réactions, des goûts et des choix dans l'échange avec les autres.

\section{Géométrie, enfant et arts graphiques}

Zerbato (2009) a constaté que « la plupart du temps dans les écoles maternelles, l'apprentissage premier de l'écriture est préparé par diverses activités motrices, visuelles et graphiques. L'objectif est d'installer des prérequis supposés pouvoir être réinvestis au cours de l'activité d'écriture. Des exercices sur fiches photocopiées sont proposés aux élèves pour leur permettre de mettre en œuvre des automatismes grapho-moteurs nécessaires à la maîtrise de l'écriture cursive. L'écriture est une activité graphique centrée sur le langage, la combinaison codée d'un système de signes».

Dès lors, apprendre à écrire, c'est apprendre un geste contrôlé visuellement qui permet le guidage sur un espace maîtrisé. Cela nécessite un apprentissage rigoureux et systématique. Chez l'enfant, tout commence par la reproduction des formes usuelles comme le cercle, le carré et le triangle aux lignes ouvertes et fermées dont le dessin révèle des apparences similaires aux silhouettes humaines. La description des lignes droites, courbes, brisées, etc., complète la gamme des instruments élémentaires à l'apprentissage de l'écriture et de la lecture. 
De ce point de vue, quelles sont les interprétations et les valeurs qui sous-tendent la compréhension de ces formes géométriques ? En effet, il existe trois formes géométriques primaires qui sont à l'origine de toutes les autres:

- $\quad$ Le carré possède quatre côtés de même mesure qui lui impriment un caractère de soliditéetde stabilité. Il se déploie dans l'espace verticalement et horizontalement. La répétition de cette forme sur une surface crée un effet d'occupation décorative de damier et de pavage. Les formes géométriques basées sur les lignes horizontales et verticales appartiennent à la famille des carrés, croix, rectangles, etc.

- Le triangle, avec ses trois côtés et ses angles aigus, est une forme vive, piquante et acide. Posé sur la pointe, il est très instable. Posé sur son plus grand côté, il est synonyme d'élévation symétrique vers le sommet du triangle. Les formes avec des lignes diagonales appartiennent à la famille du triangle, du losange, du trapèze, du zigzag, etc.

- $\quad$ Le cercle est le mouvement par excellence. Il tourne et roule sur luimême dans un mouvement continu. Symbole de la douceur et de la concentration, c'est la forme de l'univers et de l'infini sans limites. Naturellement décoratives, les formes ovoïdes, circulaires et sinusoïdales appartiennent à la famille des cercles, (in La Peinture, Giboulet \& Barilleau, 1997).

Ces modèles de formes sont choisis de manière privilégiée dans des répertoires culturels existants ou repérés dans des motifs observés dans l'environnement. L'écriture étant une activité graphique, elle permet de dessiner des traits, des lignes, des lettres de l'alphabet français qui sont des formes géométriques.Une activité graphique avec des formes géométriques peut constituer un moyen efficace pour l'apprentissage de l'écriture et de la lecture.

\section{Interdisciplinarité}

L'enfant est par nature créateur, seuls peuvent le contester ceux qui n'ont jamais observé un enfant dans ses jeux libres ou ceux qui ne l'ont découvert que déjà marqué par l'endurance d'un environnement contraignant.

L'activité de l'adulte est d'entretenir, celle de l'enfant est de développer toutes choses entières tendant vers la construction de soi. C'est précisément, par ce besoin vital de grandir tout tendu vers l'avenir et le dépassement personnel que prend son véritable sens biologique : l'activité créatrice de l'enfant. La capacité d'enchaînement et d'automatisation du geste doit être travaillée dès l'école maternelle pour espérer installer avant la fin de l'école élémentaire, l'écriture naturelle et fluide qui fait encore défaut à bon nombre d'écoliers. 
Les artsgraphiques contribuent à l'éducation esthétique : la réalisation de divers travaux bien orientés offre à l'enfant des activités de recherche, d'expérimentation, de découverte et de création, autant d'activités artistiques qui s'adressent au besoin de créer par lequel l'homme se prolonge et se réalise dans une œuvre matérielle.

Selon Gloton (1962), «les arts graphiques participent à la promotion de l'éducation physique par l'habileté du geste, la coordination motrice, la maîtrise de soi qu'ils favorisent. L'expérience vitale de l'homme est une suite d'engagements, le dessin est l'un des vecteurs du témoignage et du symbole de l'énergie humaine. Il vivifie l'expérience humaine».

Artsgraphiques et Géométrie sont liés à travers les âges par l'un de nos cinq sens. Que l'on étudie une figure géométrique ou une œuvre d'art, c'est à notre vue que nous faisons appel pour regarder, voir, observer, percevoir, contempler, admirer et décrire des compétences que nous retrouvons dans les programmes de géométrie et de l'histoire de l'art. La géométrie est génératrice de formes. De plus, tout comme la géométrie, les arts-graphiques développent la maitrise du geste et touchent de près les notions d'espace et de formes.

\section{Conclusion et suggestions}

Apprendre à réaliser des tracés ordinaires est une activité spécifique à l'école élémentaire, surtout aux cours préparatoires, à la fois originale et traditionnelle et qui possède ses propres finalités. Cette activité est en étroite liaison avec les artsgraphiques, mais aussi avec la connaissance des formes géométriques qui font référence à des domaines comme l'exploration des formes oula représentation de l'espace.

Les apprentissages fondamentaux et graphiques ont pour principaux objectifs, le développement de l'activité perceptive, l'éducation de la motricité fine, l'exploration d'une multitude d'organisations spatiales. Ceuxci se traduisent par la découverte puis la reproduction de toutes sortes de lignes, de formes, de motifs qui se structurent et s'organisent sur différents types de supports.

Au cours de la scolarité, le geste s'affermit, l'inventivité se développe et joue avec les contrastes, les couleurs, les combinaisons et les agencements divers. L'enseignant doit donc penser à choisir la nature des activités graphiques, non pas directement en fonction de la forme visée, mais en fonction des compétences grapho-motrices qu'il souhaite faire exercer. Son objectif est de veiller au développement des processus qui permettent la réalisation des tracés sans pour autant conditionner prématurément les gestes aux règles d'usage de ces écritures.

La géométrie est d'abord un procédé commode pour décorer une surface. Elle donne une précision plus grande au dessin des formes, permet 
d'organiser logiquement le décor, d'arranger avec plus de rigueur les éléments utilisés. Elle apporte l'ordre, la mesure, la pondération, l'équilibre, la noblesse. «Le carré, le cercle et le triangle sont des moyens qui possèdent en eux-mêmes, une expression plastique suffisante», écrit Kandinsky (1912).

Ainsi, Artgraphiques et Géométrie des formes apparaissent donc comme deux disciplines complémentaires pouvant contribuer de façon efficace à l'apprentissage de l'écriture et de la lecture des enfants de l'école élémentaire, quand on sait que ces deux activités de type transversal sont indispensables à leur formation intellectuelle.

\section{References:}

1. Albertil, B. (1983). Encyclopédie Universalis, Corpus7, Paris.

2. Boutonnier, J. (1976). Le dessin des enfants, SCARABEE, Paris.

3. Fontanel, B. (2008). De toutes les formes, les formes dans l'art, Collection « Mon Premier musée », Ed. PALETTE, Paris.

4. Giboulet, M. \& Barilleau, M. (1997). LA peinture, NATHAN, Paris.

5. Gloton, R. \& Clerot, C. (1971). Activités créatrices chez l'enfant, 3ème édition CASTERMAN, Paris. http://peysseri.perso.neuf.fr/PE2005/DC4.pdf: Mathématiques et arts visuels: formes géométriques ou œuvres d'art? Mémoire PE2.

6. Itten, J. (1975). L'art de la couleur, DESSAIN-TOLRA, Paris.

7. Lagoutte, D. (1990). Enseigner les arts-plastiques, ARMAND COLIN, Paris.

8. Morin, N. \& Bellocq, G. (2002). Maths et Art: rigueur et/ou flou mathématique, SCEREN-CRDP Poitou-Charentes, Paris.

9. Revue, JDI. (2005). Géométrie, une nouvelle dynamique, NATHAN, Paris.

10. Sauboa, J. (1967). Initiation aux arts-plastiques, BORDAS, Paris. 